\title{
Sirt2-associated transcriptome modifications in cisplatin-induced neuronal injury
}

\author{
Xin Zhao ${ }^{1,2}$, Wuying Du', Manchao Zhang ${ }^{1}$, Zainab O. Atiq ${ }^{1}$ and Fen Xia ${ }^{1 *}$
}

\begin{abstract}
Background: Chemotherapy-induced peripheral neuropathy is not only one of the most common causes of dose reduction or discontinuation of cancer treatment, but it can also permanently decrease the quality of life of cancer patients and survivors. Notably, Sirt2 protects many organs from various injuries, including diabetic peripheral neuropathy. As demonstrated previously by our laboratory and others, the overexpression of Sirt2 can improve cisplatin-induced neuropathy, although the mechanism is still unclear.
\end{abstract}

Results: In this study, the underlying mechanism by which Sirt2 protects neurons from cisplatin-induced injury was explored using the RNAseq technique in cultured rodent neurons. Sirt2 status was modified by genetic knockout (Sirt2/KO) and was then reconstituted in Sirt2/KO cells (Sirt2/Res). We observed 323 upregulated genes and 277 downregulated genes in Sirt2-expressing cells (Sirt2/Res) compared to Sirt2-deficient cells (Sirt2/KO). Pathway analysis suggested that Sirt2 may affect several pathways, such as MAPK, TNF, and cytokine-cytokine interaction. Furthermore, cisplatin-induced changes to the transcriptome are strongly associated with Sirt2 status. Cisplatin induced distinctive transcriptome changes for 227 genes in Sirt2-expressing cells and for 783 genes in Sirt2-deficient cells, while changes in only 138 of these genes were independent of Sirt2 status. Interestingly, changes in the p53 pathway, ECM-receptor interactions, and cytokine-cytokine receptor interactions were induced by cisplatin only in Sirt2-deficient cells.

Conclusions: This study demonstrated that Sirt2 regulates the transcriptome in cultured rodent neuronal cells. Furthermore, Sirt2-associated transcriptome regulation may be an important mechanism underlying the role of Sirt2 in organ protection, such as in cisplatin-induced neuronal injury. Sirt2 may be a potential target for the prevention and treatment of chemotherapy-induced neuropathy.

Keywords: RNAseq, Cisplatin, Neuropathy, Bioinformatics, Sirt2

\section{Background}

Cisplatin is a common and effective chemotherapeutic agent used to treat many types of cancers. Peripheral neuropathy during cisplatin treatment is a common, significant, and dose-limiting side effect [1]. This cisplatininduced neuropathy not only significantly impacts the patient's quality of life, but also impedes cancer control. According to a survey of patients treated with cisplatin, non-symptomatic and symptomatic neuropathy could be found in 38 and $28 \%$ of patients with non-

\footnotetext{
* Correspondence: FXia@uams.edu

'Department of Radiation Oncology, University of Arkansas for Medical Sciences, Little Rock, AR 72205, USA

Full list of author information is available at the end of the article
}

seminomatous testicular cancer, respectively, and 6\% were found to have disabling polyneuropathy [2]. In rodent models, cisplatin-induced neuropathy presents with mechanical allodynia, spontaneous pain, and numbness $[3,4]$.

One of the mechanistic models for cisplatin-induced peripheral neuropathy is dorsal root ganglion (DRG) sensory nerve injury $[5,6]$. It has been suggested that mitochondrial dysfunction, in particular the abnormality of axonal mitochondria $[7,8]$; changes in sensory neuron signaling cascades; calcium homeostasis; and oxidative stress may contribute to cisplatin-induced neuropathy [1]. However, the underlying cause and pathological mechanism of cisplatin-induced neuropathy is still unclear. 
As a member of the sirtuin family of proteins, Sirt2 functions as an $\mathrm{NAD}^{+}$-dependent deacetylase. Sirt2 expression is higher in olfactory sensory neurons than in neighboring cells; when compared with Sirt1, Sirt2 expression was higher in injured retinal ganglion cells in the optic nerve $[9,10]$. Additionally, Sirt2 was recently found to be a required target for neurite outgrowth. Knockdown of Sirt2 resulted in a deficiency in the LKB$1 /$ AMPK/PGC- $1 \alpha$ pathway and subsequent mitochondrial dysfunction and peripheral neuropathy in type 1 diabetic rodents [11]. Low expression of Sirt2 was also found in the diabetic cortex, which induced oxidative stress and diabetic neuropathy [12]. Furthermore, expression quantitative trait loci were used to demonstrate that Sirt2 is associated with thermal nociception [13]. Consistent with previous reports that Sirt2 overexpression could alleviate neuropathic pain in the chronic constriction injury model via deacetylation of nuclear factor-kappa B (NF-kB) [14], our recent work also demonstrates that Sirt2 protects against cisplatin-induced neuropathy in mice (manuscript submitted).

While there is a substantial amount of literature indicating a protective role for Sirt2 in cisplatin-induced neuropathy, the mechanism by which Sirt 2 protects against cisplatin-induced neuropathy is unknown. Whether or not Sirt2 is involved in regulating the transcriptome has yet to be determined. In this study, we explored the underlying mechanism of Sirt2 in protecting neurons from cisplatin-induced injury using RNAseq in cultured rodent neurons.

\section{Results}

\section{Summary of RNAseq}

To explore the underlying molecular mechanisms involved in Sirt2 function in cisplatin-induced peripheral neuropathy, we studied the Sirt2-associated and cisplatin-induced differential gene expression profiles using RNAseq data analysis in cultured neuronal cells derived from rat DRG sensory neurons. Eighteen samples were divided into 6 groups according to genotype and treatment, and each experiment was performed in triplicate (Genotype: Sirt2 genetic knockout (Sirt2/KO) and was then reconstituted in Sirt2/KO cells (Sirt2/Res), Sirt2 normal expressed (Sirt2/Ctrl); Treatment: cisplatin or Phosphate-buffered saline. After removing the barcode, index, and adapters, approximately 25.21 million reads could be detected in each sample (Fig. 1a). The remaining 14 samples were used for further analysis.

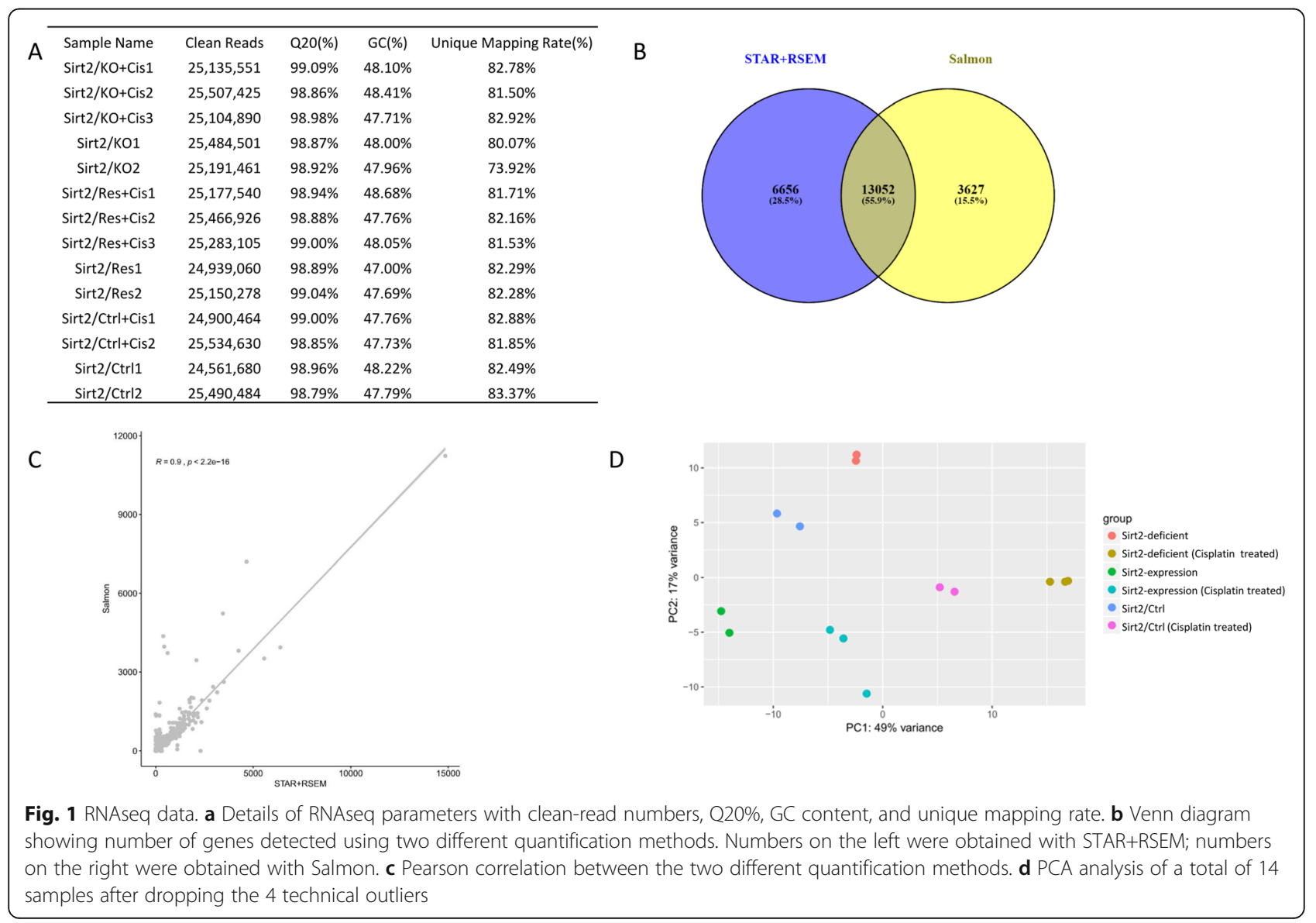


Alignment results from STAR+RSEM showed that the unique mapping rate was $81.55 \%$ on average, and 19,708 genes could be found when compared against the rat reference genome RGSC 5.0/rn5. We also used Salmon for the non-alignment quantification, and 28,421 transcripts and 16,679 genes were identified in this test. We found 13,052 overlapping genes between these two quantification tools (Fig. 1b). The technical repeats of Sirt $2 / \mathrm{Ctrl}+\mathrm{Cis}$ and Sirt2/Ctrl were analyzed to see which method was most effective, and the results showed that all three repeats had a Pearson's correlation coefficient of $r>0.99$ in both methods. A correlation of $r=0.9$ and $P<1 \times 10^{-16}$ was also found between these two different methods (Fig. 1c). Based on this, we used the overlapping genes for the analysis that followed. The results of principal component analysis showed that no extra subcluster of other group was found (Fig. 1d).

\section{Differential gene expression correlates with Sirt2 status} To increase the reliability of the analyzed results, two differential expression algorithms, edgeR and DEseq2, were used. With the cutoff false-discovery rate $($ FDR $)<$ 0.1 and fold change $>2,604$ genes showed significant change of mRNA expression using DEseq2, and 841 genes showed significance using EdgeR; 600 genes showed significance in both methods and would be used for further analysis (Fig. 2a). Among these 600 significantly genes with expression changes, 323 genes were upregulated and 277 were downregulated in Sirt2-expressing cells compared to Sirt2-deficient cells (Fig. 2b, Fig. 2c). With the cutoff of FDR $<0.1$ and fold change $>$ 10 , we found 30 genes upregulated and 25 genes downregulated in Sirt2-expressing cells when compared to Sirt2-deficient cells (Additional files 2).

To confirm Sirt2 status in cultured rodent neuron cells, we used qPCR and western blot. Levels of Sirt2 mRNA expression could still be detected in knockout cells, despite being 12.55-fold lower than levels in Sirt2expressing cells (Fig. 2d). This may be due to a residual cell population in which Sirt2 knockout was unsuccessful. Next, we used qPCR to verify the results of the algorithm. Three of the genes with the highest fold change and 4 of the genes with the lowest fold change were tested in 2 differentiated neuronal cell lines: 50B11 and PC12. With the fold change larger than 2, we verified 6 out of 7 genes in 50B11 and 5 out of 7 genes in PC12 (Table 1).

\section{Differential signaling pathways associated with Sirt2 status}

Using ClueGo from Cytoscape, we next analyzed the 600 genes whose change of expression identified as significantly

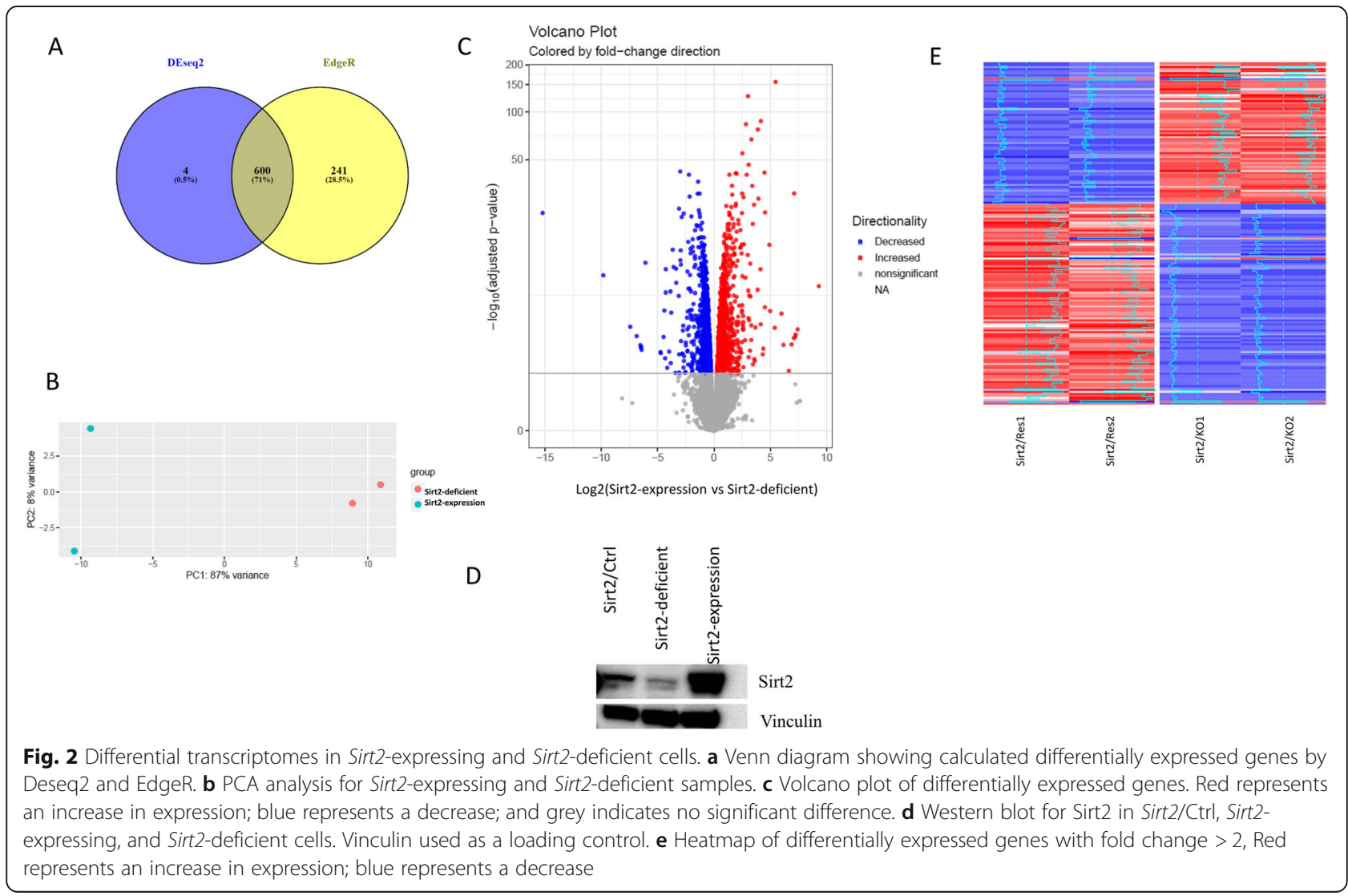


Table 1 Fold change by qPCR among different cell lines

\begin{tabular}{llll}
\hline Symbol & $\begin{array}{l}\text { Res/KO } \\
\text { RNAseq (FC) }\end{array}$ & $\begin{array}{l}\text { Res/KO } \\
50 \mathrm{~b} 11(\mathrm{FC})\end{array}$ & $\begin{array}{l}\text { Res/KO } \\
\mathrm{PC12}(\mathrm{FC})\end{array}$ \\
\hline Bmp7 & 67.75 & $2.10^{*}$ & $3.21^{*}$ \\
Mycn & 63.48 & $2.11^{*}$ & 1.08 \\
Igfbp5 & 44.27 & 0.85 & 1.37 \\
Abcc9 & 0.01 & $0.23^{*}$ & $0.24^{*}$ \\
Kcnj8 & 0.01 & $0.46^{*}$ & $0.1^{*}$ \\
Tceal3 & 0.01 & $0.40^{*}$ & $3.96^{*}$ \\
Agtr2 & 0.001 & $0.11^{*}$ & $2.39^{*}$ \\
\hline
\end{tabular}

FC fold change. All genes were normalized to Gapdh. FC $>2$ or $<0.5$ considered significant. ${ }^{*} P<0.05$

associated with Sirt2 status (Fig. 2a). We observed more than 20 pathways that were significantly affected between Sirt2-expressing and Sirt2-deficient cells (Fig. 3); these pathways include MAPK $\left(P=6.84 \times 10^{-05}\right)$, TNF $(P=1.08 \times$ $\left.10^{-04}\right)$, fluid shear stress and atherosclerosis $\left(P=1.19 \times 10^{-}\right.$ $\left.{ }^{04}\right)$, proteoglycans in cancer $\left(P=2.20 \times 10^{-04}\right)$, and cytokine-cytokine receptor interaction $\left(P=4.54 \times 10^{-04}\right)$.

\section{Sirt2 influences the transcriptome following cisplatin treatment}

We analyzed the effects of Sirt2 on gene expression in response to cisplatin in Sirt2-expressing and Sirt2-deficient 50B11 cells. With the cutoff of fold change $>2$ and FDR < 0.1 , we found that cisplatin treatment in Sirt2-expressing cells upregulated 29 genes and downregulated 58 genes. In contrast, cisplatin treatment in Sirt2-deficient cells upregulated 528 genes and downregulated 115 genes. There were 138 genes induced by cisplatin independent of Sirt2 status. To verify the analyzed results of the algorithm above, we used qPCR to examine the expression of 9 genes in Sirt2-expressing cells (3 upregulated genes and 6 downregulated genes); 7 out of 9 cisplatin-induced genes were verified in Sirt2-expressing cells, and 8 out of 9 genes were verified in Sirt2-deficient cells (Table 2).

\section{Cisplatin-induced differential signaling pathways which are associated with Sirt2 status}

Pathway analysis showed a significant difference of cisplatin-induced pathways dependent on Sirt2 status (Fig. 4). Fewer pathways were induced by cisplatin in Sirt2-expressing cells than in Sirt2-deficient cells. Furthermore, the following pathways were induced only in Sirt2-deficient cells: p53, ECM-receptor interaction, and cytokine-cytokine receptor interaction pathways. Consistent with this, we also observed increased expression of downstream targets of p53 (Fig. 5), including GADD45, GTSE1(B99), FAS, PIDD, TP53I3, SERPINE1(PAI), TSAP6, and MDM2.

\section{Discussion}

Cisplatin-induced neuropathy poses a major clinical challenge in cancer treatment. Much evidence suggests

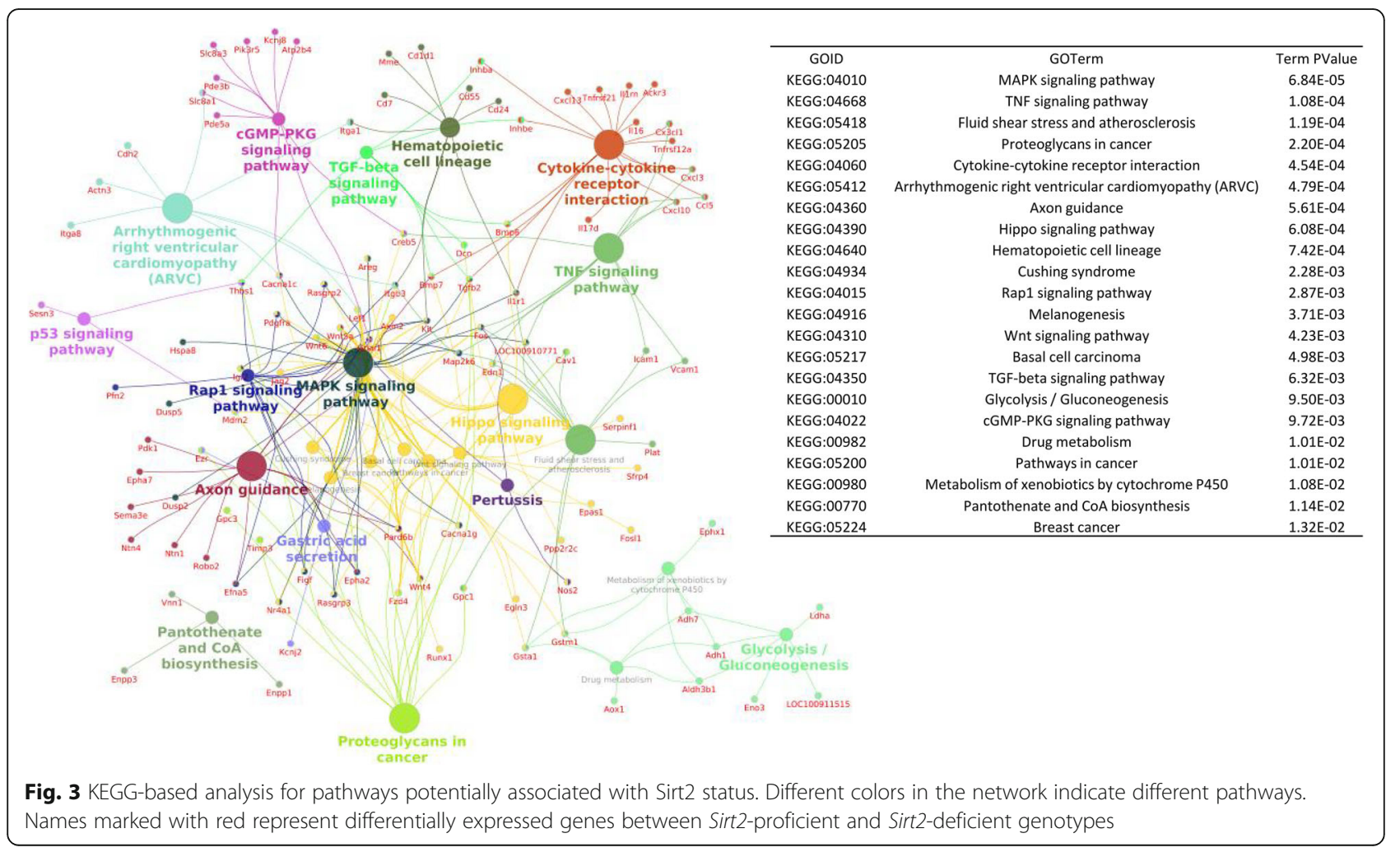


Table 2 Fold change by qPCR of cisplatin-induced transcriptome in association with Sirt2

\begin{tabular}{|c|c|c|c|c|}
\hline \multirow[t]{2}{*}{ Symbol } & \multicolumn{2}{|c|}{ Sirt2-expressing (treated vs untreated) } & \multicolumn{2}{|c|}{ Sirt2-deficient (treated vs untreated) } \\
\hline & RNAseq (FC) & $\mathrm{qPCR}(\mathrm{FC})$ & RNAseq (FC) & $\mathrm{qPCR}(\mathrm{FC})$ \\
\hline KIhdc8a & 10.09 & $2.59^{*}$ & 5.33 & $2.47^{*}$ \\
\hline Bmp7 & 5.22 & $2.57^{*}$ & 3.15 & $2.30^{*}$ \\
\hline p21 & 3.43 & $5.23^{*}$ & 5.15 & $5.45^{*}$ \\
\hline Mycn & 0.88 & 0.72 & 0.32 & $0.47^{*}$ \\
\hline lgfbp5 & 0.47 & $0.38^{*}$ & 0.68 & $0.45^{*}$ \\
\hline$A b c c 9$ & 0.44 & $0.38^{*}$ & 0.45 & $0.29^{*}$ \\
\hline Slc2a13 & 0.32 & $0.48^{*}$ & 0.25 & $0.37^{*}$ \\
\hline Tceal3 & 0.22 & 0.63 & 2.02 & $1.94^{*}$ \\
\hline Kcnj8 & 0.153 & $0.36^{*}$ & 7.63 & 1.69 \\
\hline
\end{tabular}

FC Fold change. Untreated Sirt2-expressing and Sirt2-deficient were used as a reference, respectively. All genes were normalized to Gapdh. FC $>2$ or $<0.5$ considered significant. $* P<0.05$

that Sirt2 protects tissues from injury, including neuropathy resulting from various insults. The underlying mechanism by which Sirt2 protects neurons from cisplatin-induced neuropathy is not clearly understood. In this study, we used RNAseq to examine differentially expressed genes in rodent neuronal cells with various Sirt2 genotypes in the presence and absence of cisplatin. There was a noteworthy effect of Sirt 2 on the transcriptome and various signaling pathways. We observed 323 upregulated genes and 277 downregulated genes in
Sirt2-expressing cells (Sirt2/Res) compared to Sirt2-deficient cells (Sirt2/KO). Pathway analysis suggests that Sirt2 may affect several pathways, including MAPK, TNF, and cytokine-cytokine interaction. Furthermore, cisplatin-induced transcriptome changes are strongly associated with Sirt2 status. Cisplatin induced distinct changes to the transcriptome for 227 genes in Sirt2-expressing cells and to 783 genes in Sirt2-deficient cells, while changes in only 138 of these genes were independent of Sirt2 status. Interestingly, the following pathways

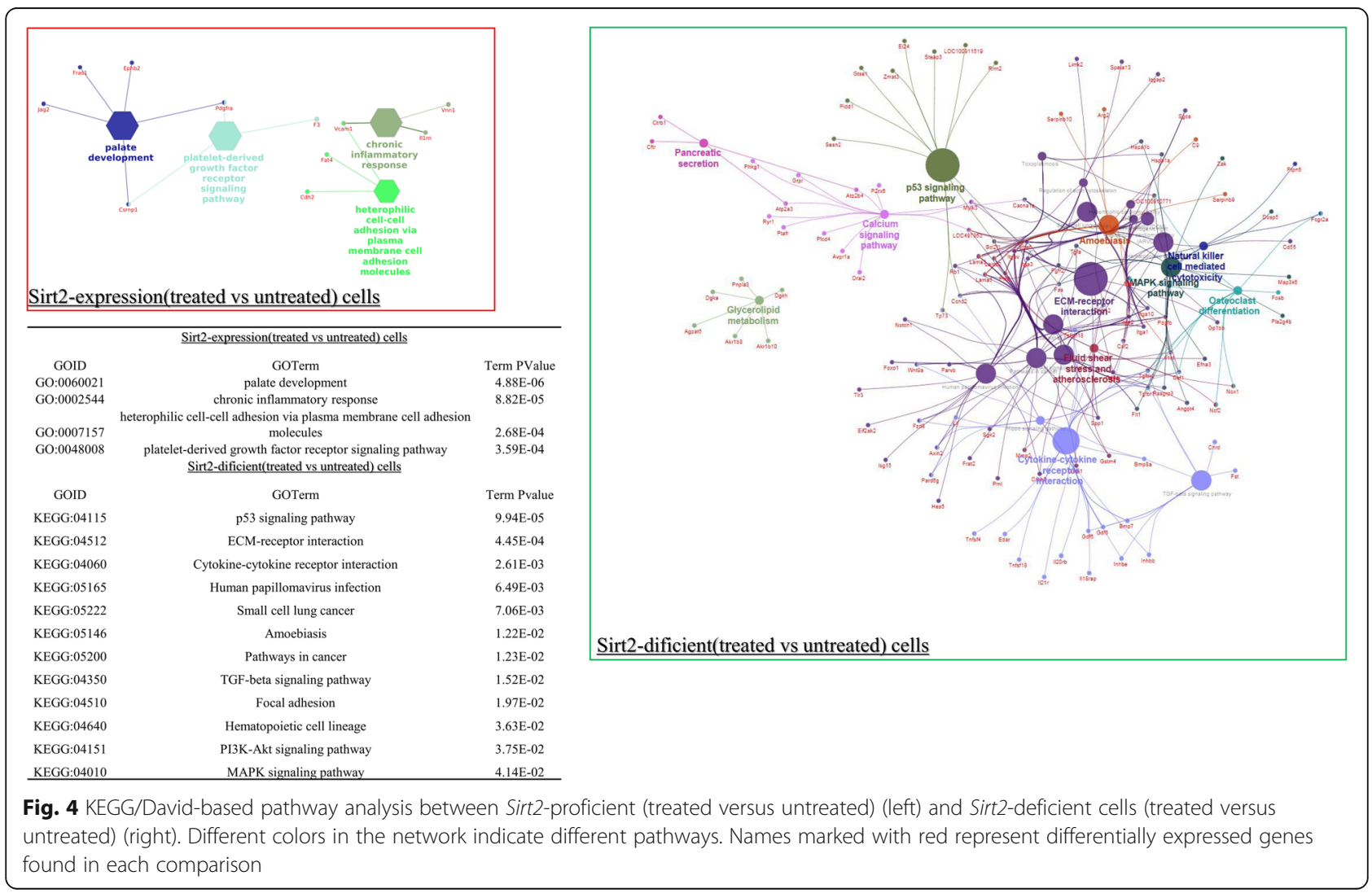




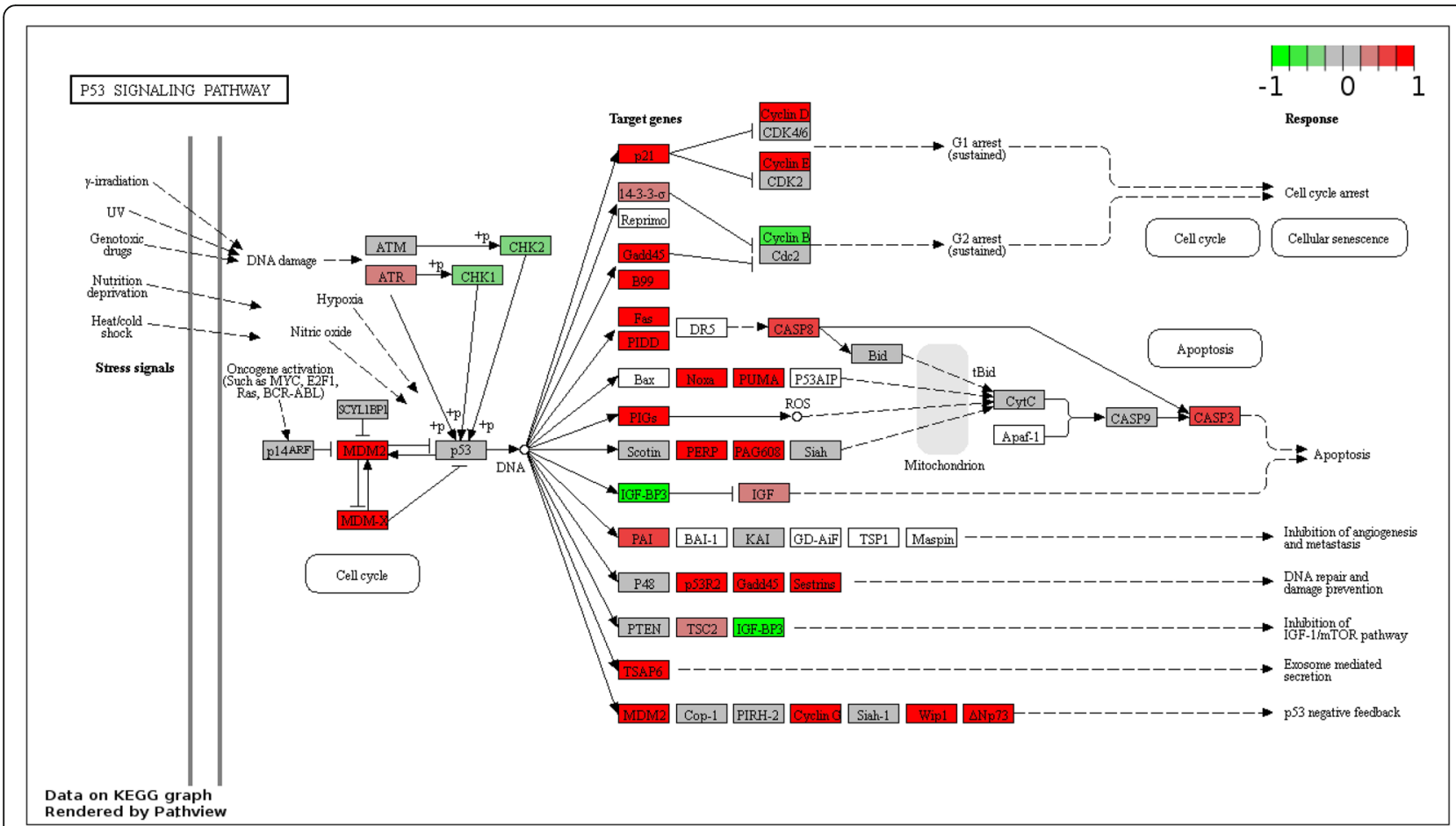

Fig. 5 Cisplatin-induced P53 signaling pathway genes are differentially expressed between Sirt2-deficient and Sirt2-proficient cells. Red indicates higher expression and green indicates lower expression when comparing between Sirt2-deficient and Sirt2-proficient cells

were induced by cisplatin only in Sirt2-deficient cells: p53, ECM-receptor interaction, and cytokine-cytokine receptor interaction.

Other genes that have been verified by $\mathrm{qPCR}$ in current study may also affect neuronal function. Studies show that overexpression of bone morphogenetic protein 7 (BMP7) is beneficial in both nerves and Schwann cells after sciatic nerve injury, and it improves neuropathy in rats [15]. The $A B C C 9$ gene and its polypeptide product SUR2 are increasingly implicated in the aged human brain and induce the hippocampal sclerosis of aging [16]. As the receptor subtype of angiotensin II, AGTR2 is expressed in sensory neurons and could be a potential target for nociception and neuronal regeneration [17]. $M Y C N$, a member of $M Y C$ proto-oncogenes, expression levels may have a positive relationship with disease-free survival in astrocytoma and meningioma [18]. Also, potassium voltage-gated channel subfamily J member 8 (KCNJ8) may help to regulate blood pressure with the help of $A B C C 9$ [19].

Sirt2 plays roles in many biological processes, including cell cycle regulation, tumorigenesis, neurodegeneration, lipid metabolism, and glucose metabolism [20]. One hypothesis is that Sirt2 regulation of cisplatininduced neuropathy is immune mediated. We showed that knocking out Sirt2 induce changes in inflammatory pathways, including MAPK, cytokine-cytokine, and TNF pathways. Twenty-six genes were enriched in these three pathways; interestingly, all of these genes were upregulated in Sirt2-expressing cells by at least 2-fold (Supplemental Table 1). Additionally, some researchers suggested that Sirt2 is involved in inflammatory pathways because Sirt2-KO mice had more morphological changes in microglia and an increase in proinflammatory cytokines in the brain [21]. Topical administration of a Sirt2-fused carrier protein to an inflamed ear reduced the levels of pro-inflammatory cytokines and inhibited the activation of NF-KB and MAPKs [22]. Additionally, another study showed that the MAPK pathway does function during platinum-induced neuropathy through the activation of p38 and ERK1/2 in DRG neurons [23].

The relationship between Sirt 2 and the calcium ion channel. The calcium signaling pathway could also explain Sirt2-related cisplatin sensitivity to some degree. Our previous proteomic data showed that Sirt2-overexpressing mice have increased protein levels of CAMK2A $(\mathrm{FC}=1.55, P=0.02)$, CAMK2D $(\mathrm{FC}=1.39, P=0.02)$, and CAMK2G $(\mathrm{FC}=1.42, P=0.03)$ when compared to SIRT2-deficient mice (manuscript submitted). We found that genes involved in calcium signaling (from KEGG) tend to be more highly expressed in Sirt2-expressing cells (Supplemental Table 2). We propose that this may be due to the impairment of the mitochondrial membrane caused by cisplatin. Mitochondrial dysfunction can lead to abnormal calcium uptake or leakage. To 
repair the harm caused by cisplatin, Sirt 2 might modify and strengthen the cisplatin-impaired calcium channel.

RNAseq is a credible method for studying global transcriptome regulation. However, a limited sample size can contribute bias to the results. Here, we performed qPCR to validate the RNAseq data. Because Sirt2 has deacetylase activity, future research should use an increased sample size, focus on acetyl-proteomes, and evaluate acetylation levels combined with multi-omics, such as proteomics and transcriptomes.

\section{Conclusions}

Using RNAseq to investigate the interaction of genotype with treatment, we demonstrated that the difference in Sirt2 expression in cultured rodent neuronal cells leads to a significant change in the transcriptome with and without cisplatin treatment. These data suggest that transcription regulation could be an important mechanism underlying the protective function of Sirt2 in cisplatin-induced neuropathy. In addition, Sirt 2 could potentially serve as a target in the treatment and prevention of cisplatin-induced neuropathy.

\section{Methods}

\section{CRISPR-Cas9 gene editing}

First, the single guide RNA (sgRNA) designed by the Atum bio CRISPR design tools, 5 pairs of sirt 2 single guide RNA oligoes had been designed for the screening. The oligoes are designed based on the target site sequence ( $20 \mathrm{bp}$ ) and needs to be flanked on the 3 ' end by a 3 bp NGG PAM sequence [24]. Lenti-CRISPR-v2 (AddGene 52,961) contains two expression cassettes, hSpCas9 and the chimeric guide RNA. The vector can be digested using BsmBI, and a pair of annealed oligoes can be cloned into the single guide RNA scaffold. Second, the cloned sgRNA Lenti-CRISPR-v2 vector sequencing by the hU6 promoter primer (5'-GAGGGCCTAT TTCCCATGATT-3') then make lentivirus. CMV-EGFP as a positive control for viral production. Lentivirus concentrated by $15,000 \mathrm{rpm}$ spin $2 \mathrm{~h}$ in $4{ }^{\circ} \mathrm{C}$ [25]. 50B11 and PC12 cells infected by the concentrated lentivirus, $48 \mathrm{~h}$ later selected by puromycin $2 \mu \mathrm{g} / \mathrm{ml}$ for another $48 \mathrm{~h}$ then harvest the cells detect the sirt 2 expression by western blot. One of the five pairs was working perfect on rat SIRT2 gene (5'-GCGGAAGTCAGGGATTCC TG-3').

Cell culture, neuronal differentiation, and drug treatment Cells were cultured at the University of Arkansas for Medical Sciences. 50B11 cells were from Dr. Youwei Zhang of Case Western Reserve University and PC12 (CRL-1721) was purchased from ATCC. Cultures were treated as follows: 50B11 cells were maintained in Dulbecco's Modified Eagle's medium (DMEM) supplemented with 10\% FBS,
$0.2 \mathrm{M}$ glutamine, $1 \times \mathrm{B}-27$ supplement, $0.2 \%$ glucose, and $1 \%$ penicillin/streptomycin/glutamine and incubated at $37^{\circ} \mathrm{C}$ with $5 \% \mathrm{CO}_{2}$ [26]. The cells were cultured for at least $24 \mathrm{~h}$, and confluence was allowed to reach $70 \%$ before differentiation. Forskolin $(25 \mathrm{mM})$ was added directly to the culture medium for a final concentration of $75 \mu \mathrm{M}$. Full differentiation of neurite outgrowth appeared after 12 h.

PC12 cells were maintained in Dulbecco's Modified Eagle's medium (DMEM) supplemented with 5\% calf serum, $5 \%$ horse serum and $1 \%$ penicillin/streptomycin/ glutamine and incubated at $37^{\circ} \mathrm{C}$ with $5 \% \mathrm{CO}_{2} .1 \%$ nerve growth factor was used for the cell differentiation. Cells were feed every other day with differentiating medium until ready for experiment.

Cisplatin was added to the culture medium at a final concentration of $2 \mu \mathrm{g} / \mathrm{ml}$. After the $24 \mathrm{~h}$, cells were harvested for RNA extraction. A flow chart of this process is shown in Supplemental Figure 1.

\section{RNA extraction}

RNA was extracted according to TRIzol guidelines. All cells were lysed and homogenized by TRIzol and temporarily stored at $-80^{\circ} \mathrm{C}$; then, $0.3 \mathrm{ml}$ chloroform was added per $1 \mathrm{ml}$ of TRIzol reagent. The samples were mixed vigorously and centrifuged at $12,000 \times \mathrm{g}$ for $15 \mathrm{~min}$ at $4{ }^{\circ} \mathrm{C}$. RNA precipitated from the aqueous phase was mixed with $0.5 \mathrm{ml}$ of isopropanol and centrifuged at 12 , $000 \times \mathrm{g}$ for $30 \mathrm{~min}$. The supernatant was removed, and $0.5 \mathrm{ml} 75 \%$ ethanol was used for washing. The RNA concentration was determined with a NanoDrop One spectrophotometer.

\section{RNAseq and alignment}

All RNA samples passed the threshold of quality control by the Agilent 2100 Bioanalyzer. The library preparation and RNAseq were processed at BGI-Hong Kong. The cDNA was synthesized and sheared into a 250-bp fragment, end-repaired, dA-tailed, and adaptor-ligated. After a 4-cycle PCR program, the libraries were sequenced on the BGI-SEQ500 platform with the 50-bp single-end sequencing strategy. Eighteen samples were divided into 6 groups with 3 repeats according to genotype and treatment, and 25 million reads were obtained for each sample (on average). All samples passed the quality control (QC) threshold of Q20 > 98\%. After the first PCA and cluster analysis, 4 samples (1 Sirt2/Res, 1 Sirt2/KO, 1 Sirt2/Ctrl, and 1 Sirt $2 / \mathrm{Ctrl}+\mathrm{Cis})$ were eliminated as outliers. Fourteen samples that passed initial QC were used for further analysis (Fig. 1a). RGSC 5.0/rn5 RefSeq was downloaded from UCSC Genome Browser (http://genome.ucsc.edu). We used STAR 2.6.1b for the alignment [27]. FeatureCounts from the GATK4.0.8.1 package was used for the quantification [28]. RSEM 1.3.1 was used 
for the Transcripts Per Million (TPM) calculation with the parameters of "--star --estimate-rspd --appendnames --output-genome-bam.” Salmon 0.11.3 was also used for the quantification of TPM with the parameters of "quant -i -1 A --gcBias" [29]. The entirety of the pipeline can be seen in Supplemental Figure 2.

\section{Differentially expressed genes and pathway analysis}

To analyze differentially expressed genes among the different genotypes and treatment conditions, we used DEseq2 and edgeR [30, 31]. During the procedure, the $P$-value (two-tailed) was calculated using a two-sample t-test. Corrections for false-positives (type I errors) were performed using Benjamin's false discovery rate (FDR). We used "FDR $<0.1$, Fold Change $>2$ or $<0.5$ " as the threshold to judge the significance of differences in gene expression between the different conditions. All of the procedures were under the environment of $\mathrm{R}$ 3.4.3. Pathway analysis was performed using DAVID (http://david. abcc.ncifcrf.gov/), Gene Ontology (http://geneontology. org/), or the Kyoto Encyclopedia of Genes and Genomes (https://www.genome.jp/kegg/pathway.html). A hypergeometric test with the Benjamin and Hochberg false discovery rate (FDR) was performed using the default parameters to adjust the $P$-value. Cytoscape v3.6.1 and Pathview were used for pathway visualization $[32,33]$.

\section{Real-time quantitative PCR (qPCR)}

The results of the RNAseq algorithm were verified by real-time quantitative PCR (qPCR, TaqMan). The top differentially expressed genes between Sirt2-proficient and Sirt2-deficient cells were chosen for verification. All TaqMan assays were purchased from ThermoFisher (Abcc9, Rn00464842_m1; Bmp7, Rn01528889_m1; Mycn, Rn01473353_m1; Igfbp5, Mm00516037_m1; Tceal3, Mm02747701_Sh; Kcnj8, Rn01492857_m1; Agtr2, Rn00560677_s1; and Gapdh, Rn01775763_g1). The TaqMan procedure was performed using an ABI StepOne system with 96 -well plates as follows: $50^{\circ} \mathrm{C}$ for $2 \mathrm{~min}$ to pre-heat the mixture, $95^{\circ} \mathrm{C}$ for $20 \mathrm{~s}$ to activate the Taq polymerase, 40 cycles of $95^{\circ} \mathrm{C}$ for $1 \mathrm{~s}$, and $60^{\circ} \mathrm{C}$ for $20 \mathrm{~s}$. The qPCR data were analyzed with $\mathrm{R}$ 3.4.3. An unpaired t-test was performed to compare delta cycle threshold $(\mathrm{dCt})$ values obtained in different groups. The data are expressed as relative fold-change based on the $2^{-\Delta \Delta C t}$ method [34].

\section{Availability of data and materials}

Raw sequence data were deposited at the European $\mathrm{Nu}$ cleotide Archive (ENA) (http://www.ebi.ac.uk/ena/data/ view/PRJEB36706).

\section{Supplementary information}

Supplementary information accompanies this paper at https://doi.org/10. 1186/s12864-020-6584-2.

\section{Additional file 1: Supplemental Figure 1. Timeline of cell} differentiation. Samples were divided into 6 groups (Sirt2/Res, Sirt2/Ctrl, Sirt2/KO, Sirt2/Res + Cis, Sirt2/Ctrl+Cis, or Sirt2/KO + Cis). Cells were differentiated with Forskolin for $12 \mathrm{~h}$ and treated with cisplatin for another $24 \mathrm{~h}$. After that, RNA was harvested. Supplemental Figure 2. Project pipeline. First, adapters and barcodes were removed to obtain clean data. Second, Salmon and STAR+RSEM, two methods for quantification, were used. Third, after quantification, gene Venn diagram, PCA analysis, correlation stat, cluster analysis, and differential expressed analysis (by using DEseq2 and EdgeR together) were performed. Finally, pathway analysis was performed. Supplemental Table 1. MAPK pathway-related genes significantly differentially expressed between Sirt2expressing cells and Sirt2-deficient cells. Supplemental Table 2. Calcium pathway-related genes significantly differentially expressed between Sirt2expressing cells and Sirt2-deficient cells.

Additional File 2 Potential targets of deferential expressed genes with fold change $>10$ and FDR $<0.1$ in comparisons of Sirt2-expressing cells vs Sirt2-deficient cells; Sirt2-expression (cisplatin treated vs untreated) cells and Sirt2-deficient (cisplatin treated vs untreated) cells.

\section{Abbreviations}

ABCC9: ATP-binding cassette subfamily C member 9; AGTR2: Angiotensin II receptor type 2; BMP7: Bone morphogenetic protein 7; CAMK2A: Calcium/ calmodulin dependent protein kinase II alpha; CAMK2D: Calcium/calmodulin dependent protein kinase II delta; CAMK2G: Calcium/calmodulin dependent protein kinase II gamma; Ctrl: Control; FC: Fold change; FDR: False discovery rate; KCNJ8: Potassium voltage-gated channel subfamily J member 8; KO: Knockout; PCA: Principal component analysis; QC: Quality control; Res: Rescue; Sirt2: Sirtuin 2; TPM: Transcripts per million

\section{Acknowledgements}

Not applicable.

\section{Authors' contributions}

Research idea conception: XZ and FX RNA Extraction: XZ and WD CRISPRCas9 gene editing and Cell culture: XZ, WD and MZ Analytical approach design: X Z and FX Data analysis: XZ Manuscript writing: XZ, ZA and FX Manuscript discussion and revision: $X Z, Z A$ and FX. All authors read and approved the final manuscript.

\section{Funding}

This study was supported by NIH grants R01 CA188500, and R01 CA163838 to F.X. The funding bodies played no role in the design of the study and collection, analysis, and interpretation of data and in writing the manuscript.

\section{Availability of data and materials}

The datasets supporting the conclusions of this article are included within the article (and its additional files).

Ethics approval and consent to participate Not applicable.

\section{Consent for publication}

Not applicable.

\section{Competing interests}

The authors declare that they have no competing interests.

\section{Author details}

'Department of Radiation Oncology, University of Arkansas for Medical Sciences, Little Rock, AR 72205, USA. ${ }^{2}$ Institute for Systems Genetics, NYU Langone Health, New York, NY 10016, USA. 
Received: 18 March 2019 Accepted: 17 February 2020

Published online: 02 March 2020

\section{References}

1. Starobova H, Vetter I. Pathophysiology of chemotherapy-induced peripheral neuropathy. Front Mol Neurosci. 2017;10:174.

2. Strumberg D, Brugge S, Korn MW, Koeppen S, Ranft J, Scheiber G, Reiners C, Mockel C, Seeber S, Scheulen ME. Evaluation of long-term toxicity in patients after cisplatin-based chemotherapy for non-seminomatous testicular cancer. Ann Oncol. 2002;13(2):229-36.

3. Park HJ, Stokes JA, Pirie E, Skahen J, Shtaerman Y, Yaksh TL. Persistent hyperalgesia in the cisplatin-treated mouse as defined by threshold measures, the conditioned place preference paradigm, and changes in dorsal root ganglia activated transcription factor 3: the effects of gabapentin, ketorolac, and etanercept. Anesth Analg. 2013;116(1):224-31.

4. Krukowski K, Nijboer CH, Huo X, Kavelaars A, Heijnen CJ. Prevention of chemotherapy-induced peripheral neuropathy by the small-molecule inhibitor pifithrin-mu. Pain. 2015;156(11):2184-92

5. Meijer C, de Vries EG, Marmiroli P, Tredici G, Frattola L, Cavaletti G. Cisplatininduced DNA-platination in experimental dorsal root ganglia neuronopathy. Neurotoxicology. 1999;20(6):883-7.

6. Zamble DB, Lippard SJ. Cisplatin and DNA repair in cancer chemotherapy. Trends Biochem Sci. 1995;20(10):435-9.

7. Flatters SJ, Bennett GJ. Studies of peripheral sensory nerves in paclitaxelinduced painful peripheral neuropathy: evidence for mitochondrial dysfunction. Pain. 2006:122(3):245-57.

8. Zheng $H$, Xiao WH, Bennett GJ. Functional deficits in peripheral nerve mitochondria in rats with paclitaxel- and oxaliplatin-evoked painful peripheral neuropathy. Exp Neurol. 2011;232(2):154-61.

9. Yu TT, Mclntyre JC, Bose SC, Hardin D, Owen MC, McClintock TS. Differentially expressed transcripts from phenotypically identified olfactory sensory neurons. J Comp Neurol. 2005;483(3):251-62.

10. Geng Y, Wang J, Liang J, Xu C, Zhi Y. Expression of Sirt1 and Sirt2 in injured optic retina of calorie restricted rats. Eye Sci. 2011;26(4):221-4.

11. Schartner E, Sabbir MG, Saleh A, Silva RV, Roy Chowdhury S, Smith DR, Fernyhough P. High glucose concentration suppresses a SIRT2 regulated pathway that enhances neurite outgrowth in cultured adult sensory neurons. Exp Neurol. 2018;309:134-47.

12. Roy Chowdhury S, Djordjevic J, Thomson E, Smith DR, Albensi BC Fernyhough $\mathrm{P}$. Depressed mitochondrial function and electron transport complex II-mediated $\mathrm{H} 2 \mathrm{O} 2$ production in the cortex of type 1 diabetic rodents. Mol Cell Neurosci. 2018;90:49-59.

13. Bryant CD, Bagdas D, Goldberg LR, Khalefa T, Reed ER, Kirkpatrick SL, Kelliher JC, Chen MM, Johnson WE, Mulligan MK, et al. C57BL/6 substrain differences in inflammatory and neuropathic nociception and genetic mapping of a major quantitative trait locus underlying acute thermal nociception. Mol Pain. 2019;15:1744806918825046.

14. Zhang Y, Chi D. Overexpression of SIRT2 alleviates neuropathic pain and Neuroinflammation through Deacetylation of transcription factor nuclear factor-kappa B. Inflammation. 2018:41(2):569-78.

15. Tsai MJ, Pan HA, Liou DY, Weng CF, Hoffer BJ, Cheng H. Adenoviral gene transfer of bone morphogenetic protein-7 enhances functional recovery after sciatic nerve injury in rats. Gene Ther. 2010;17(10):1214-24.

16. Nelson PT, Jicha GA, Wang WX, Ighodaro E, Artiushin S, Nichols CG, Fardo DW. ABCC9/SUR2 in the brain: Implications for hippocampal sclerosis of aging and a potential therapeutic target. Ageing Res Rev. 2015;24(Pt B):11125.

17. Anand U, Facer $P$, Yiangou $Y$, Sinisi M, Fox M, McCarthy T, Bountra C, Korchev YE, Anand $P$. Angiotensin II type 2 receptor (AT2 R) localization and antagonist-mediated inhibition of capsaicin responses and neurite outgrowth in human and rat sensory neurons. Eur J Pain. 2013;17(7):101226.

18. Estiar MA, Javan F, Zekri A, Mehrazin M, Mehdipour P. Prognostic significance of MYCN gene amplification and protein expression in primary brain tumors: astrocytoma and meningioma. Cancer Biomark. 2017;19(3): 341-51

19. Ellis JA, Lamantia A, Chavez R, Scurrah KJ, Nichols CG, Harrap SB. Genes controlling postural changes in blood pressure: comprehensive association analysis of ATP-sensitive potassium channel genes KCNJ8 and ABCC9. Physiol Genomics. 2010;40(3):184-8.
20. Gomes P, Fleming Outeiro T, Cavadas C. Emerging role of Sirtuin 2 in the regulation of mammalian metabolism. Trends Pharmacol Sci. 2015;36(11): 756-68.

21. Pais TF, Szego EM, Marques $O$, Miller-Fleming $L$, Antas $P$, Guerreiro $P$, de Oliveira RM, Kasapoglu B, Outeiro TF. The NAD-dependent deacetylase sirtuin 2 is a suppressor of microglial activation and brain inflammation. EMBO J. 2013;32(19):2603-16.

22. Kim MJ, Kim DW, Park JH, Kim SJ, Lee CH, Yong Jl, Ryu EJ, Cho SB, Yeo HJ, Hyeon J, et al. PEP-1-SIRT2 inhibits inflammatory response and oxidative stress-induced cell death via expression of antioxidant enzymes in murine macrophages. Free Radic Biol Med. 2013;63:432-45.

23. Rutkove SB. Effects of temperature on neuromuscular electrophysiology. Muscle Nerve. 2001;24(7):867-82.

24. Mao Y, Zhang Z, Feng Z, Wei P, Zhang H, Botella JR, Zhu JK. Development of germ-line-specific CRISPR-Cas9 systems to improve the production of heritable gene modifications in Arabidopsis. Plant Biotechnol J. 2016;14(2): 519-32.

25. Liu Y, Liu F, Yu H, Zhao X, Sashida G, Deblasio A, Harr M, She QB, Chen Z, Lin HK, et al. Akt phosphorylates the transcriptional repressor bmi1 to block its effects on the tumor-suppressing ink4a-arf locus. Sci Signal. 2012;5(247): ra77.

26. Chen W, Mi R, Haughey N, Oz M, Hoke A. Immortalization and characterization of a nociceptive dorsal root ganglion sensory neuronal line. J Peripher Nerv Syst. 2007;12(2):121-30.

27. Dobin A, Davis CA, Schlesinger F, Drenkow J, Zaleski C, Jha S, Batut P, Chaisson M, Gingeras TR. STAR: ultrafast universal RNA-seq aligner. Bioinformatics. 2013;29(1):15-21.

28. McKenna A, Hanna M, Banks E, Sivachenko A, Cibulskis K, Kernytsky A, Garimella K, Altshuler D, Gabriel S, Daly M, et al. The genome analysis toolkit: a MapReduce framework for analyzing next-generation DNA sequencing data. Genome Res. 2010;20(9):1297-303.

29. Patro R, Duggal G, Love MI, Irizarry RA, Kingsford C. Salmon provides fast and bias-aware quantification of transcript expression. Nat Methods. 2017; 14(4):417-9

30. Robinson MD, McCarthy DJ, Smyth GK. edgeR: a bioconductor package for differential expression analysis of digital gene expression data. Bioinformatics. 2010:26(1):139-40.

31. Love MI, Huber W, Anders S. Moderated estimation of fold change and dispersion for RNA-seq data with DESeq2. Genome Biol. 2014;15(12):550.

32. Shannon P, Markiel A, Ozier O, Baliga NS, Wang JT, Ramage D, Amin N, Schwikowski B, Ideker T. Cytoscape: a software environment for integrated models of biomolecular interaction networks. Genome Res. 2003;13(11): 2498-504.

33. Luo W, Brouwer C. Pathview: an R/bioconductor package for pathwaybased data integration and visualization. Bioinformatics. 2013;29(14):1830-1.

34. Livak KJ, Schmittgen TD. Analysis of relative gene expression data using real-time quantitative PCR and the 2 (-Delta Delta $C(T))$ method. Methods. 2001;25(4):402-8.

\section{Publisher's Note}

Springer Nature remains neutral with regard to jurisdictional claims in published maps and institutional affiliations.

Ready to submit your research? Choose BMC and benefit from

- fast, convenient online submission

- thorough peer review by experienced researchers in your field

- rapid publication on acceptance

- support for research data, including large and complex data types

- gold Open Access which fosters wider collaboration and increased citations

- maximum visibility for your research: over $100 \mathrm{M}$ website views per year

At $\mathrm{BMC}$, research is always in progress.

Learn more biomedcentral.com/submission 